\title{
Tecnicas de mantenimiento
}

\section{Maintenance techniques}

FERNANDEZ-GOMEZ, Tomas $\dagger^{*}$, RAMIREZ-RODRIGUEZ, Ramón Rodolfo, MIRANDASANCHEZ, Francisco Javier y MERINO-ROSAS, Ignacio Celestino

Tecnológico Nacional de México/Instituto Tecnológico de Orizaba, Departamento de Metal-Mecánica, Ote 9 Num 1852, Col E. Zapata C.P 94320, Orizaba, Veracruz, México

ID $1^{\text {er }}$ Autor: Tomas, Fernande- Gomez / ORC ID: 0000-0002-031, Researcher ID Thomson: fernandez_gt, CVU CONACYT ID: 65262

ID 1 er $^{\text {er }}$ Coautor: Ramon Rodolfo, Ramirez-Rodriguez / ORC ID: 0000-0002-272, Researcher ID Thomson: Y6fbnMXGYG5Aee4GKCZWDmGjz7TuztXq, CVU CONACYT ID: 1024679

ID $2^{\text {do }}$ Coautor: Francisco Javier, Miranda-Sanchez / ORC ID: 0000-0001-711, Researcher ID Thomson: qQeu179m1C0dMYjrGocgNKdk3mTImeaz, CVU CONACYT ID: 781882

ID $3^{\text {er }}$ Coautor: Ignacio Celestino, Merino-Rosas / ORC ID: 0000-0002-558, Researcher ID Thomson: ubnBf1CDjZwU8xp1kWIJUg0jhcsCArje, CVU CONACYT ID: IT18D309

DOI: $10.35429 / J I E .2019 .8 .3 .6 .13$

Recibido 04 de Abril, 2019, Aceptado, 03 de Junio, 2019

\section{Resumen}

Las mejoras enfocadas son actividades en las que se desarrollan con la intervención de las diferentes áreas en un proceso productivo, es un desarrollo en el proceso de mejora continua similar al existente en procesos de Control Total de Calidad aplicando procedimientos y técnicas de mantenimiento. El mantenimiento es un conjunto de actividades que se realizan diariamente en los equipos, incluyendo inspección, lubricación, limpieza, intervenciones menores, cambio de herramientas y piezas estudiando posibles mejoras. El mantenimiento progresivo es un pilar de lo más importante en la búsqueda de beneficios en una organización industrial. El mantenimiento de calidad busca establecer como propósito que en el equipo tenga el "cero defectos". La PTEE o Productividad Total Efectiva de los Equipos es una medida de la productividad real de los equipos, se trata de una medida que indica la cantidad de tiempo utilizado por los equipos, efectividad global del equipo evalúa el rendimiento del equipo mientras está en funcionamiento, esto permite priorizar entre varios proyectos de manteamiento, aquellos más significativos en la mejora de la planta.

Planificación, Mantenimiento, Productividad

\begin{abstract}
The focused improvements are activities in which they are developed with the intervention of the different areas in a productive process, it is a development in the process of continuous improvement similar to that existing in processes of Total Quality Control applying procedures and maintenance techniques. Maintenance is a set of activities that are carried out daily in the equipment, including inspection, lubrication, cleaning, minor interventions, change of tools and pieces studying possible improvements. Progressive maintenance is a pillar of the most important in the pursuit of benefits in an industrial organization. The maintenance of quality seeks to establish as a purpose that the team has the "zero defects". The PTEE or Total Effective Productivity of the Equipment is a measure of the real productivity of the equipment, it is a measure that indicates the amount of time used by the equipment, the overall effectiveness of the equipment evaluates the performance of the equipment while it is in operation, this allows to prioritize between several maintenance projects, those most significant in the improvement of the plant.
\end{abstract}

Planning, Maintenance, Productivity

Citación: FERNANDEZ-GOMEZ, Tomas, RAMIREZ-RODRIGUEZ, Ramón Rodolfo, MIRANDA-SANCHEZ, Francisco Javier y MERINO-ROSAS, Ignacio Celestino. Técnicas de mantenimiento. Revista de Ingeniería Industrial. 2019 3-8: 6-13

\footnotetext{
* Correspondencia del Autor (correo electrónico: fernandez_gt@yahoo.com)

$\uparrow$ Investigador contribuyendo como primer autor.
} 


\section{Introducción}

La aparición de fallos y averías en los componentes de una instalación industrial trae consigo la disminución de los beneficios que pudieran derivarse del proceso productivo en cuestión, aquellas averías que dan lugar a la indisponibilidad del proceso provocan una merma de ingresos $\mathrm{y}$, asimismo, originan un incremento de los costes de producción, ya que, como mínimo, habrá que reparar o sustituir el equipo averiado, En los tiempos actuales, caracterizados por un creciente grado de competencia en la totalidad de los mercados que provoca la erosión de los márgenes comerciales, el aseguramiento de la capacidad productiva se configura como un factor fundamental para el mantenimiento o mejora de la rentabilidad asociada a una instalación o proceso industrial.

El objetivo de este trabajo es aportar ideas y plantear como se llevará a cabo el pilar de mantenimiento productivo total, se presentarán los aspectos más significativos de la metodología TPM (Total Productive Maintenance), considerada como una herramienta muy importante a implantar en una instalación industrial que contribuya a la mejora de la productividad $\mathrm{y}$, por consiguiente, al incremento de la rentabilidad de los procesos implicados y del valor de los activos invertidos.

\section{Desarrollo}

Normalmente en las industrias se presta mucha atención a secciones que se encuentran emparentadas con el producto y normalmente la sección mantenimiento queda relegada a un plano inferior. ¿Por qué?, muy sencillo; se tiene la idea errónea de que los procesos que llevan a buen puerto el procesamiento de un producto dependen exclusivamente de producción. los procesos industriales dependen más que nada del buen funcionamiento de los equipos a afectados al mismo. Sencillamente "las máquinas nos dan de comer. Ahora bien; ¿Quién tiene la responsabilidad de hacer funcionar las máquinas de manera óptima?; la respuesta es mantenimiento.

\section{¿Qué es TPM?}

Mantenimiento Productivo Total es la traducción de TPM (Total Productive Maintenance).
El TPM es el sistema japonés de mantenimiento industrial desarrollado a partir del concepto de "mantenimiento preventivo" creado en la industria de los Estados Unidos.

Asumimos el término TPM con los siguientes enfoques: la letra "M" representa acciones de management y mantenimiento. Es un enfoque de realizar actividades de dirección y transformación de empresa.

La letra " P" está vinculada a la palabra "productivo" o "productividad de equipos asociar a un término con una visión más amplia como "perfeccionamiento" La letra "T" de la palabra "total" se interpreta como "todas las actividades que realizan toda la persona que trabajan en la empresa.

Los conceptos que se describen posteriormente se jerarquizan en términos de la influencia de unos en otros, se puede afirmar que el mantenimiento, en sus variantes de preventivo y correctivo, influye demasiado en el resto de los elementos de la confiabilidad de un dispositivo.

\section{Pilar 1 Mejoras Enfocadas (Kobetsu Kaizen)}

Las mejoras enfocadas son actividades en las que se desarrollan con la intervención de las diferentes áreas comprometidas en el proceso productivo, esto con el objetivo de maximizar toda efectividad en los equipos, procesos $\mathrm{y}$ plantas.

Esto conlleva al desarrollo del proceso de mejora continua que es muy similar al existente en los procesos de Control Total de Calidad aplicando procedimientos y técnicas de mantenimiento.

Las técnicas TPM ayudan a eliminar dramáticamente las averías de los equipos. El procedimiento seguido para realizar acciones de mejoras enfocadas sigue los pasos del conocido Ciclo Deming o PHVA (Planificar-HacerVerificar-Actuar),

El desarrollo de las actividades Kobetsu Kaizen se realizan a través de los pasos mostrados en la figura 


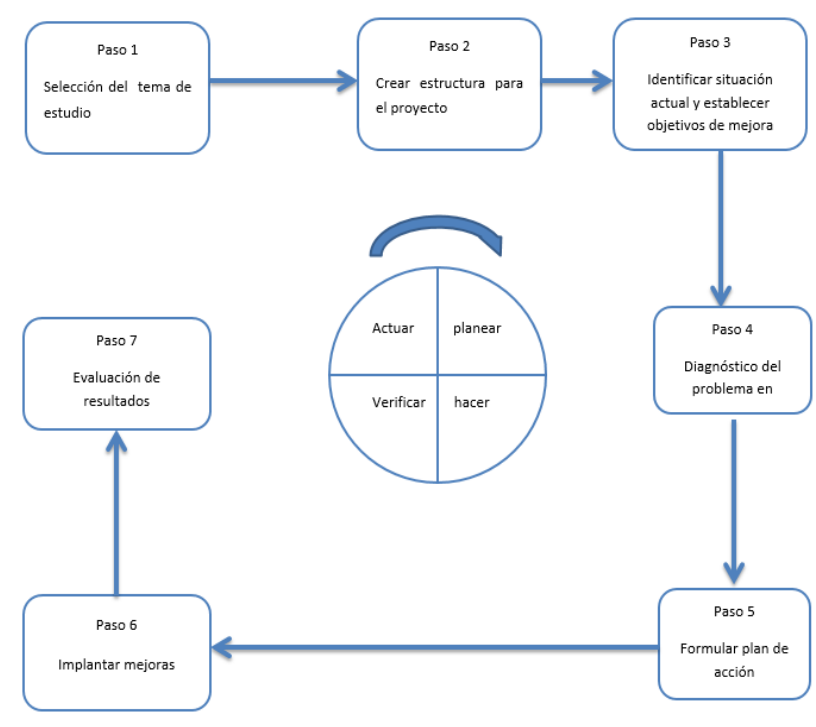

Figura 1 Desarrollo de actividades

Paso 1: Seleccionar un tema de estudio

Esto se puede seleccionar empleando diferentes criterios

- Objetivos superiores de la dirección
industrial
- $\quad \begin{aligned} & \text { Problemas de calidad y entregas al } \\ & \text { cliente }\end{aligned}$
- $\quad \begin{aligned} & \text { Relación con procesos de mejora } \\ & \text { continua }\end{aligned}$
-
Factores innovadores y otros

Paso 2: Crear la estructura para el proyecto

Frecuentemente se utiliza los del equipo interfuncional. Esta clase de equipos intervienen trabajadores de diferentes áreas que son directamente involucrados en el proceso productivo como supervisores, operadores, personal técnico de mantenimiento, compras o almacenes proyectos, ingeniería de proceso y control de calidad

Paso 3: Identificar la situación actual y formular objetivos

En este paso analizaremos el problema en forma general y se identificaran las perdidas principales asociadas con el problema seleccionado. En esta fase se debe recoger o procesar la información sobre averías, fallos, reparaciones y otras estadísticas sobre las pérdidas por problemas de calidad, energía, análisis de capacidad de proceso y de los tiempos de operación para identificar los cuellos de botella, paradas, etc.
Esta información se debe presentar en forma gráfica y Estratificada para facilitar su interpretación y el diagnóstico del problema.

Paso 4: Diagnostico del problema

Antes de utilizar alguna técnica analítica para estudiar y solucionar el problema se deben establecer y mantener las condiciones básicas que aseguren el funcionamiento apropiado del equipo. Estas condiciones básicas incluyen: limpieza, lubricación, chequeos de rutina, apriete de tuercas, etc. Esto implica realizar actividades de mantenimiento autónomo en las áreas seleccionadas como piloto para la realización de las mejoras enfocadas.

Paso 5: Formular plan de acción

Una vez concluida la investigación y analizado las diferentes raíces del problema, se establecerá un plan de acción para la eliminación de las causas críticas. A partir de las propuestas se establecen las actividades y tareas señaladas necesarias para lograr los objetivos formulados.

Paso 6: Implantar mejoras

Una vez planificadas las acciones con detalle se procede a implantarlas. Es importante durante la implantación de las acciones contar con la participación de todas las personas involucradas en el proyecto incluyendo el personal operador.

Las mejoras no deben ser impuestas ya que si se imponen por orden superior no contarán con un respaldo total del personal operativo involucrado. Cuando se pretenda mejorar los métodos de trabajo, se debe consultar y tener en cuenta las opiniones del personal que directa o indirectamente intervienen en el proceso.

Paso 7: Evaluar los resultados

Es muy importante que los resultados obtenidos en una mejora sean publicados en una cartelera o paneles, en toda la empresa lo cual ayudará a asegurar que cada área se beneficie de la experiencia de los grupos de mejora. El comité u oficina encargada de coordinar el TPM debe llevar un gráfico o cuadro en la cual se controlen todos los proyectos, y garantizar que todos los beneficios y mejoras se mantengan en el tiempo 


\section{Pilar 2 Mantenimiento autónomo}

El mantenimiento autónomo está compuesto por un conjunto de actividades que se realiza diariamente por todos los trabajadores en los equipos que operan, incluyendo inspección, lubricación, limpieza, intervenciones menores, cambio de herramientas y piezas estudiando posibles mejoras, analizando y solucionando problemas del equipo y acciones que conduzcan a mantener el equipo en las mejores condiciones de funcionamiento. Una de las visiones modernas en mantenimiento desarrollar a través del Mantenimiento Autónomo.

El Mantenimiento Autónomo implica un cambio cultural en la empresa, especialmente en el concepto: "yo fabrico y tu conserva el equipo", en lugar de "yo cuido mi equipo". Para lograrlo es necesario incrementar el conocimiento que poseen los operarios para lograr un total dominio de los equipos.

Esto implica desarrollar las siguientes capacidades en los operarios. lo convierte en una estrategia poderosa de transformación continua de empresa.

Sirve para adaptar permanentemente a la organización hacia las nuevas exigencias del mercado y para crear capacidades competitivas centradas en el c conocimiento que las estacar es la creación de un trabajo disciplinado y respetuoso de las normas procedimientos.

\section{Pilar 3 Mantenimiento progresivo o planificado}

El mantenimiento progresivo es uno de los pilares más importantes en la búsqueda de beneficios en una organización industrial. El $\Lambda \mathrm{PM}$ le ha dado a este pilar el nombre de Mantenimiento Planificado (MP).

Consideramos que el término MP puede comunicar mejor el propósito de este pilar, que consiste en la necesidad de avanzar gradualmente hacia la búsqueda de la meta "cero averías" para una planta. Ver siguiente figura

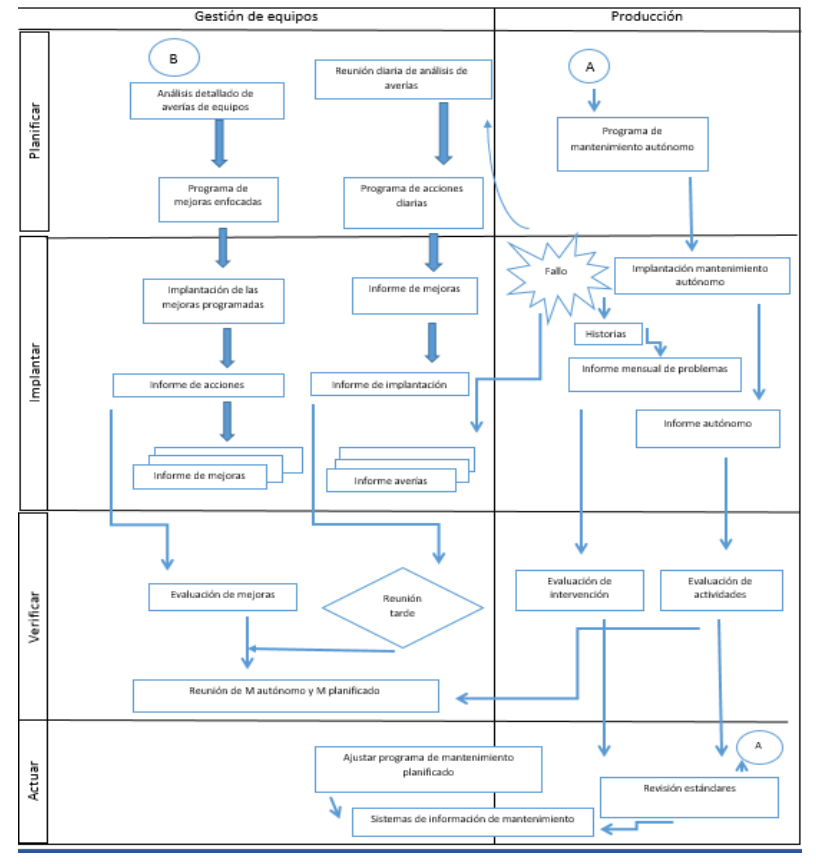

Figura 2 Relación entre las acciones de mantenimiento y producción

\section{Limitaciones de los enfoques tradicionales de mantenimiento planificado}

El mantenimiento planificado que se practica en numerosas empresas presenta los siguientes aspectos:

- No se dispone de información histórica necesaria para establecer el tiempo más adecuado para realizar las acciones de mantenimiento preventivo. Los tiempos son establecidos de acuerdo con la experiencia, recomendaciones de fabricante $y$ otros criterios con poco fundamento técnico y sin el apoyo en datos e información histórica sobre comportamiento pasado.

- Se aprovecha la parada de un equipo para "hacer todo lo necesario en la máquina" ya que la tenemos disponible. ¿Será necesario un tiempo similar de intervención para todos los elementos y sistemas de un equipo?, será esto económico?.

- Se aplican planes de mantenimiento preventivo a equipos que poseen un alto deterioro acumulado. Este deterioro afecta la dispersión de la distribución (estadística) de fallos, imposibilitando la identificación de un comportamiento regular del fallo y con el que se debería establecer el plan de mantenimiento.

- $\quad$ Propósito estratégico.

- $\quad$ Responsabilidad recíproca.

FERNANDEZ-GOMEZ, Tomas, RAMIREZ-RODRIGUEZ, Ramón Rodolfo, MIRANDA-SANCHEZ, Francisco Javier y MERINO-ROSAS, Ignacio Celestino. Técnicas de mantenimiento. Revista de Ingeniería Industrial. 2019 


\section{Propósito estratégico}

Son ambiciones a las que aspira la organización. El propósito estratégico tiene presente la visión de cómo debe ser la posición de liderazgo deseada de la empresa, el camino y las pautas para establecer criterios que la organización utilizará para el progreso.

- $\quad$ El propósito estratégico es un reto que en esta situación se aprecia en todo tipo de empresas e inclusive en aquellas que poseen certificaciones y programas o modelos de calidad avanzados.

- $\quad$ El trabajo de mantenimiento planificado no incluye acciones Kaizen para la mejora de los métodos de trabajo. No se incluyen acciones que permitan mejorar la capacidad técnica y mejora de la fiabilidad del trabajo de mantenimiento, como tampoco es frecuente observar el desarrollo de planes para eliminar la necesidad de acciones de mantenimiento. Esta también debe ser considerada como una actividad de mantenimiento preventivo.

\section{Aportes del TPM a la mejora de MP}

El TPM posee una mayor óptica o visión del proceso de gestión preventiva de equipos y utiliza tres grandes estrategias:

1. Actividades para prevenir y corregir a verías en equipos a través de rutinas diarias periódicas y predictivas.

2. Actividades Kaizen orientadas a mejorar las características de los equipos

Actividades Kaizen para mejorar la competencia administrativa y técnica de la función mantenimiento

Si se comparan las dos estrategias anteriores sugeridas dentro del TPM con las prácticas habituales de mantenimiento planificado, observamos que existe una diferencia significativa en cuanto al alcance de sus actividades.

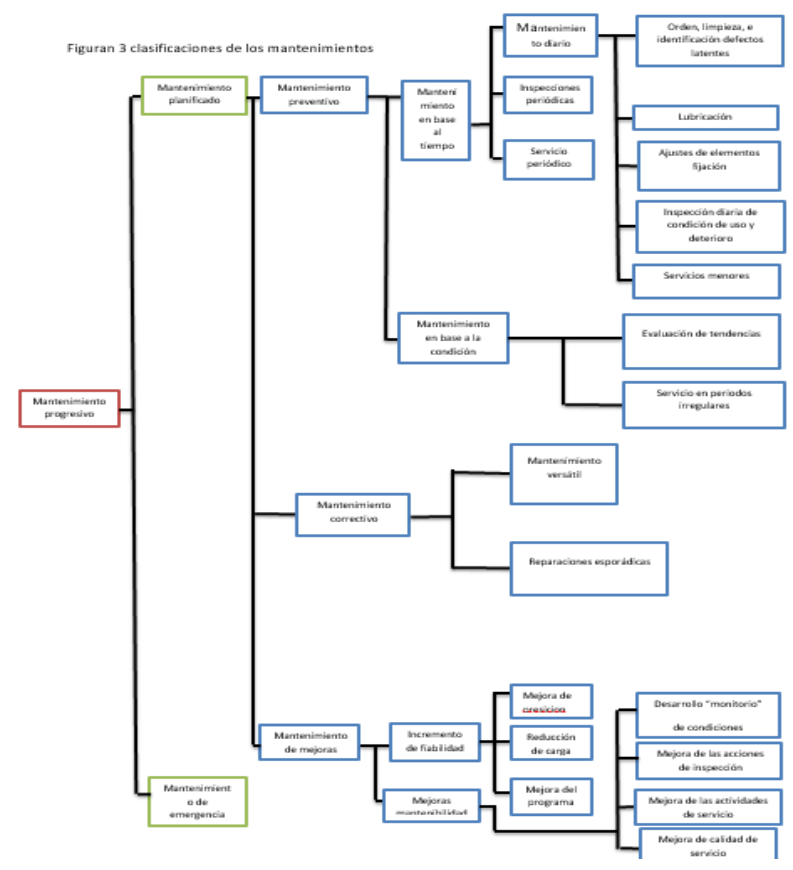

Figura 3 Clasificación de los métodos de mantenimiento

\section{Pilar 4 Mantenimiento de Calidad}

Tiene como propósito establecer las condiciones del equipo en un punto donde el "cero defectos" es factible. Las acciones del MC buscan verificar y medir las condiciones "cero defectos" regularmente con el objeto de facilitar la operación de los equipos en la situación donde no se generen defectos de calidad. Mantenimiento de calidad no es...

- Aplicar técnicas de control de calidad a las tareas de mantenimiento.

- Aplicar un sistema ISO a la función de mantenimiento.

- Utilizar técnicas de control estadístico de calidad al mantenimiento.

- Aplicar acciones de mejora continua a la función de mantenimiento.

Mantenimiento de Calidad es...

- Realizar acciones de mantenimiento orientadas al cuidado del equipo para que este no genere defectos de calidad.

- $\quad$ Prevenir defectos de calidad certificando que la maquinaria cumple las condiciones para "cero defectos" y que estas se encuentran dentro de los estándares técnicos.

Observa las variaciones de las características de los equipos para prevenir defectos y tomar acciones adelantándose a la situación de anormalidad potencial. 
- Realizar estudios de ingeniería del equipo para identificar los elementos del equipo que tienen una alta incidencia en las características de calidad del producto final, realizar el control de estos elementos de la máquina e intervenir estos elementos.

\section{$>\quad$ Etapas del pilar MC}

El JIPM (Japan Institute of Plant Maintenance), ha establecido nueve etapas para el desarrollo del MC. Estas se deben auditar y siguen las estrategias de prueba piloto, equipo modelo y transferencia del conocimiento utilizados en otros pilares TPM.

- $\quad$ Etapa 1: Identificación de la situación actual del equipo.

- $\quad$ Etapa 2: Investigación de la forma como se generan los defectos.

- $\quad$ Etapa 3: Identificación y análisis de las condiciones 3M (Materiales, Máquina y Mano de obra).

- $\quad$ Etapa 4: Estudiar las acciones correctivas para eliminar "fugas".

- Etapa 5: Analizar las condiciones del equipo para productos sin defectos $\mathrm{y}$ comparar los resultados.

- Etapa 6: Realizar acciones Kaizen o de mejora de las condiciones $3 \mathrm{M}$ de Toyota.

- Etapa 7: Definir las condiciones y estándares de las 3M.

- Etapa 8: Reforzar el método de inspección.

- Etapa 9: Valorar los estándares utilizados.

\section{Gestión TPM}

Concepto de productividad total efectiva de los equipos (PTEE)

La PTEE es una medida de la productividad real de los equipos. Esta medida se obtiene multiplicando los siguientes índices: $\mathrm{PTEE}=\mathrm{AE} X \mathrm{XGE}$

AE-Aprovechamiento del equipo. Se trata de una medida que indica la cantidad del tiempo calendario utilizado por los equipos. El AE está más relacionado con decisiones directivas sobre uso del tiempo calendario disponible que con el funcionamiento en sí del equipo.
Esta medida es sensible al tiempo que habría podido funcionar el equipo, pero por diversos motivos los equipos no se programaron para producir el $100 \%$ del tiempo. Otro factor que afecta el aprovechamiento del equipo es el tiempo utilizado para realizar acciones planificadas de mantenimiento preventivo. El AE se puede interpretar como un porcentaje del tiempo calendario que ha utilizado un equipo para producir.

EGE-Efectividad Global del Equipo. Esta medida evalúa el rendimiento del equipo mientras está en funcionamiento. La EGE está fuertemente relacionada con el estado de conservación y productividad del equipo mientras está funcionando.

Una de las claves para la puesta en marcha del TPM en forma exitosa es que la dirección comunique el motivo del cambio estratégico que se inicia en los centros productivos con tanta claridad y en una forma que logre el interés en un principio y un compromiso total en todos los niveles para llevar a cabo esta estrategia. Se debe crear el suficiente entusiasmo para lograr que la puesta en práctica del TPM sea una verdadera cruzada contra todo lo que sea despilfarro en la organización. Sin embargo, no existe o es imposible contar con un menú de trayectorias para implantar con éxito la estrategia TPM en compañías occidentales. Los pasos sugeridos por el JIPM deben ser tomados como pautas concretas para abordar el trabajo. La implantación del TPM en empresas con carácter latino es la menos estudiada; la mejor evidencia de lo que se debe hacer o no se debe hacer proviene de las experiencias reportadas y de las lecciones aprendidas por los directivos y de las compañías, a continuación se presenta un resumen para el inicio de una estrategia como TPM.

Diseñar una organización con los componentes, capacidades y recursos para llevar a cabo la estrategia. El equipo directivo de un centro productivo forma el comité TPM. Cada directivo o pequeños grupos de directivos constituyen el equipo líder de cada pilar TPM. El objetivo consiste en involucrar a todos los directivos en la dirección de las acciones TPM. La coordinación de estos equipos la realiza la dirección superior del centro productivo. 
El segundo elemento organizativo es la coordinación. No es aconsejable asignar el proyecto a una sola persona de la empresa, especialmente con la interpretación de "responsable". Esta figura de un diseño organizativo deficiente puede conducir a dificultades en la realización de la estrategia TPM. Una tercera figura organizativa son los equipos de trabajo a nivel operativo. Estos equipos son los responsables de ejecutar numerosas acciones TPM.

Asignar presupuestos para el desarrollo de la estrategia TPM. Implantar TPM implica realizar acciones que requieren inversiones. Es posible que la más significativa tiene que ver con la recuperación del deterioro acumulado de los equipos de las instalaciones industriales. Si se pretende mejorar el nivel de productividad de una planta, es necesario mejorar la gestión de los equipos, mejorar el mantenimiento preventivo y esto exige inversiones que se recuperarán posteriormente con los mejores niveles de productividad y utilización de los equipos. Otro factor es la formación técnica de los niveles operativos y la mejora de la capacidad de gestión de los mandos medios y encargados

\section{Conclusión}

Los procesos industriales dependen más que nada del buen funcionamiento de los equipos afectados al mismo; ¿quién tiene la responsabilidad de hacer funcionar las máquinas de manera óptima?; En todas las empresas es mantenimiento.

El TPM es una estrategia compuesta por una serie de actividades ordenadas que una vez implantadas ayudan a mejorar la competitividad de una organización industrial o de servicios. Tiene como propósito en las acciones cotidianas que los equipos operen sin averías y fallos, eliminar toda clase de perdidas, mejorar la fiabilidad de los equipos $y$ emplear verdaderamente la capacidad industrial instalada.

El modelo original TPM propuesto por el Japan Institute of Plant Maintenance (JIPM) sugiere utilizar pilares específicos para acciones concretas diversas, las cuales se deben implantar en forma gradual y progresiva, asegurando cada paso dado mediante acciones de autocontrol del personal que interviene.

\section{Referencias}

[1] La editorial Renovetec, a través del IRIM (Instituto Renovete de Ingeniería del Mantenimiento) ha publicado la guía Técnica los recursos humanos en mantenimiento (2014)

[2] Ing. Talavera Jaime Ingeniero Mecánico, Pontificia Universidad Católica del Perú. Postgrado en Operaciones Universidad de Piura, Especialización en Gestión de Mantenimiento y Confiabilidad ASME, Participante del Sénior Management Program del IE Business School 2015 España

[3] Martínez-delgado. E Cabrera-Gómez, j., y are-castro Diagnostico del servicio de mantenimiento de grupos electrónicos de energía, ingeniería mecánica, 22.2-92-99 B. A. (2019)

[4] Enciso. P. B.. S. D. Gómez Francisco R. G. M.M Croño, C. G. Y Martin. R. G. E. (2019) Operatividad y mantenimiento. Factores clave durante el diseño del buque ingeniería naval

[5] Gestión y planificación del Mantenimiento Industrial Producido, desarrollado, editado y publicado por Integra Markets Escuela de Gestión Empresarial 2da Edición - 2018 ISBN 9781370710768

[6] Cárcel Carrasco F. Javier. (2014). la gestión del conocimiento en la ingeniería del mantenimiento industrial. Valencia, España: Omniascience

[7] Sosa Vásquez. Tomas (2014) el secreto del mantenimiento industrial. Editorial palibrio

[8] Gonzales Vícto Medrano José Mantenimiento: Técnicas y aplicaciones industriales (Español) Pasta blanda - 8 jun 2017.

[9] González García, Raimundo Heber_Mantenimiento Industrial: Organización, Control Y Gestión (Español) Pasta blanda - 1 sep 2016

[10] González Fernández Francisco Javier Teoría y Práctica del Mantenimiento Industrial Avanzado - Confemetal Categoría Ingeniería Año 2015 Idioma Español № páginas 708. 
[11] Carnero Moya María Del Carmen . Programas de Mantenimiento Predictivo Eae Editorial Academia Española Editorial Eae Editorial Academia Española Categoría Libros de textos Tema Engineering (general) Año 2012 Idioma Español $\mathrm{N}^{\circ}$ páginas 420

[12] Vilardell Eugenio Nieto Mantenimiento Industrial Práctico (Tinta Negra) (Español) Pasta blanda - 18 oct 2013

[13] García Garrido Santiago Ingeniería de Mantenimiento Manual práctico para la gestión eficaz del mantenimiento (C) Santiago García Garrido 2009-2012 Todos los derechos reservado

[14] Sánchez Gómez Ana maría técnicas de mantenimiento predictivo, metodología de la aplicación en las organizaciones 2017

[15] Olarte C., Botero William; A., Cañón Marcela; Z., BENHUR A. análisis de vibraciones una herramienta clave en el mantenimiento predictivo Scientia Et Technica, vol. XVI, núm. 45, agosto, 2010, pp. 219-222 Universidad Tecnológica de Pereira Pereira, Colombia 